ELUA

ISSN 2171-6692

Núm. 36, 2021, págs. 45-65

https://doi.org/10.14198/ELUA.2021.36.03

\title{
Aplicación de la Semántica de Marcos y la Teoría Sentido-Texto a las colocaciones en español del campo léxico de los aparatos
}

\author{
Implementation of Frame Semantics and Meaning-Text Theory to the spanish \\ collocations of the lexical field of the appliances
}

\author{
Diego SÁNCHEZ MAYOR \\ Universidad Complutense de Madrid, España \\ diesan08@ucm.es \\ https://orcid.org/0000-0002-4847-0808
}

\begin{abstract}
Resumen
Este trabajo propone una aplicación de la Semántica de Marcos y la Teoría Sentido-Texto a las colocaciones del español. Se ha señalado que algunas funciones léxicas se solapan entre ellas u ofrecen problemas de granularidad en el análisis, pues se usa una determinada función léxica para una colocación con verbos de significado poco preciso (como la bomba funcio$n a)$ y la misma función léxica para colocaciones con verbos de significado más restringido (como la bomba salta o la bomba explota). Aquí defendemos que, para solventar ese problema, se pueden enriquecer las funciones léxicas con indicaciones tomadas de los marcos semánticos. Para demostrarlo hemos creado una combinación ad hoc de funciones léxicas y marcos para un corpus de colocaciones verbonominales del campo léxico de los aparatos (entre las que se encuentran detonar la bomba,
\end{abstract}

\begin{abstract}
This study portrays an implementation of the Frame Semantics framework and the Meaning-Text Theory to some Spanish collocations. It has been pointed out that some lexical functions overlap each other or offer some granularity problems in the analysis, since a certain lexical function is used for a collocation with verbs of imprecise meaning (such as la bomba funciona [the bomb works]), and the same lexical function for collocations with verbs of more restricted meanings (such as la bomba salta [the bomb jumps], la bomba explota [the bomb explodes]). Here we support that, in order to solve this problem, the lexical functions can be enriched with indications taken from the semantic frames. To expose this, we have created and ad hoc combination of lexical functions and frames for a corpus of verb-nominal collocations of the lexical field
\end{abstract}

Para citar este artículo: Sánchez Mayor, D. (2021). Aplicación de la semántica de marcos y la teoría sentido-texto a las colocaciones en español del campo léxico de los aparatos. ELUA, (36), 45-65. https://doi.org/10.14198/ELUA.2021.36.03.

Recibido: 07/09/2021, Aceptado: 18/10/2021

(C) 2021 Diego Sánchez Mayor

Este trabajo está sujeto a una licencia de Reconocimiento 4.0 Internacional de

Creative Commons (CC BY 4.0) 
el ordenador recaba la información, reglar el radiador, desvalijar un cajero, etc.). En este trabajo describimos algunas de las colocaciones con las que hemos trabajado y mostramos cómo con nuestra propuesta se puede ampliar la granularidad del análisis. Así, por ejemplo, en el caso de la bomba funciona recurrimos al marco "Using", mientras que en el caso de la bomba explota recurrimos a "Getting_triggered". Los datos que mostramos en este trabajo señalan que la conjunción de marcos y funciones léxicas permite discriminar rasgos semánticos no detectados por las funciones léxicas aisladamente (un patrón diverso como el ordenador \{recaba / analiza / explora \} descrito bajo una misma función léxica), así como inducir categorizaciones semánticas (tipos de aparatos que caen bajo un mismo patrón colocacional: aparatos informáticos, explosivos, de indicación informativa, etc.) o incluso especificar rasgos ontológicos no señalados (diferencias existentes entre los significados de robar y saquear o entre llenar o vaciar). Concluimos defendiendo que la conjunción de ambos modelos lexicológicos permite una descripción más amplia y pormenorizada del comportamiento de las colocaciones.

PALABRAS CLAVE: colocación; función léxica (FL); marco; Semántica de Marcos (SM); Teoría Sentido-Texto (TST). of the appliances (among which can be examined detonar la bomba [detonate the bomb], el ordenador recaba la información [the computer collects the information], reglar el radiador [regulate the radiator], desvalijar un cajero [robe a cashier], etc.). Here we describe some of the collocations we have worked with and we exhibit how our proposal can improve the granularity of the analysis. Thus, for instance, in the case of the bomb works we use the "using" frame, while in the case of the bomb jumps we use "Getting triggered". The data indicates that the conjunction of frames and lexical functions makes it possible to discern semantic features not detected by the lexical function itself, such as a diverse pattern as el ordenador \{recaba / analiza / explora \} [the computer \{ collects / analyses / explores\}] described under the same lexical function. It also induce semantic categorizations (types of appliances that fall under the same collocation pattern: aparatos informáticos [computer devices], explosivos [explosives], de indicación informativa [indications], etc.) or even unmarked ontological features (differences between the meanings of robar [to steal] and saquear [to plunder], or llenar [to fill] and vaciar [to empty]). We conclude by arguing that the combination of both lexicological models allows a broader and more exhaustive description of the behaviour of collocations.

KEY WORDS: collocation; lexical function (LF); frame; Frame Semantics (FS); MeaningText Theory (MTT).

\section{INTRODUCCIÓN}

Este artículo propone un modelo de descripción válido para las colocaciones en español. En él nos preguntamos si existe alguna manera de refinar el tipo de identificación y descripción que suele realizarse para las colocaciones. Sugerimos, para la consecución de este objetivo, la conjunción de dos modelos teóricos que aporten una mayor granularidad en el análisis de los datos lingüísticos.

Para ello, recurriremos a la Semántica de Marcos (SM) y la Teoría Sentido-Texto (TST), dos modelos lexicológicos cuya compenetración defienden Bouveret y Fillmore (2008). Tomamos este trabajo como punto de partida y tratamos de validar, en la medida de nuestras posibilidades, su propuesta a través de un estudio de corpus. 


\subsection{Concepto de colocación}

La colocación, comprendida tradicionalmente como uno de los fenómenos lingüísticos más difícilmente definibles, se ajusta a aquella combinatoria sintagmática léxica que, compuesta por un mínimo de dos constituyentes gráficos, dista formalmente de otros tipos fraseológicos manifiestamente idiomáticos, como son la paremia o la locución.

No obstante, conviene advertir que el concepto de colocación con que se trabaja en este estudio, concerniente a la TST, difiere ligeramente de aquel esgrimido tradicionalmente. Puede alegarse de manera muy directa que la TST, si bien no es un modelo creado ex profeso para el estudio de las colocaciones, sí que sustenta gran parte de su labor teórica y aplicativa en este ámbito. Corpas (2003: 78), por ejemplo, comenta que, entre las dos corrientes que estudian el fenómeno colocacional, la estadística y la semántica, la TST ha supuesto un gran avance en esta segunda, en tanto en cuanto ha aportado un medio excepcional de sistematizar los vínculos semánticos existentes en estas combinaciones léxicas.

Mel'čuk (2015b), creador de la TST, confiere a la colocación el estatus de frasema, de ahí que se entienda este compuesto como un fenómeno adscrito a la fraseología. Sin embargo, es conveniente definir la noción de frasema para entender este sentido 'parcialmente fraseológico' atribuido a la colocación. Un frasema es un sintagma no libre; entendiendo por 'no libre': "si, y solo si, no puede construirse, a partir de un contenido informativo dado, de manera regular y no restringida” (Mel'čuk 2006: 14).

Esta definición invita a comprender, en apariencia, que el significado colocacional presenta un carácter no composicional, algo que no es cierto. De alguna manera, parece ser que, en las colocaciones, los dos o más elementos integrados conservan el significado, aunque no de la misma forma, dependiendo de la posición que ocupen. La adopción del concepto de semifrasema permite ilustrar aquella realidad sintagmática en que únicamente uno de los constituyentes enlazados en la colocación conserva su significado primario.

La estricta coocurrencia léxica a la que se ve impuesta el fenómeno colocacional es explicada a menudo como un caso de cohesión semántica que ciertas piezas léxicas comparten - "gang up": una especie de confabulación léxica" Polguère (2014: 406) -. No obstante, esta combinatoria tan restringida no es con la que se trabaja únicamente. La noción de colocación manejada en la TST abarca desde las combinatorias más libres o triviales (usar \{un ordenador / una batidora / un coche $\}$; encender \{la televisión / la lámpara / la estufa $\}$ ) hasta aquellas con coocurrencias más estrictas (repicar las campanas; asfixiar un motor; calar un coche; purgar un radiador; etc.).

\subsection{Metodología y sección de los datos de estudio}

Bouveret y Fillmore (2008) defienden una primera conjunción de dos modelos teóricos de corte léxico: FrameNet, la interfaz informática a través de la que se despliega la Semántica de Marcos, y la Teoría Sentido-Texto, con sus funciones léxicas, los elementos con los que poder trazar los ejes paradigmáticos y sintagmáticos del léxico. En el mencionado trabajo, los autores restringieron su estudio de colocaciones verbonominales a aquellas en las que, sirviéndose de un verbo de apoyo, permiten una forma sintética de expresión. Véanse los siguientes ejemplos (traducción propia) ${ }^{1}$ :

1 Ejemplos de Bouveret y Fillmore (2008 : 1): 1a) She gave me wise advice, 1b) She advised me wisely; 2a) Les parents sont libres de donner leur consentement ou de refuser, 2b) Les parents sont libres de consentir ou de refuser. 
(1) i. Juan dio un beso a Azucena.

i'. Juan besó a Azucena.

ii. Hice el examen y me fui.

ii'. Me examiné y me fui.

Como puede constatarse, estos enunciados disponen de alternativas con bastante relevancia dada su frecuencia. La dimensión significativa del artículo de Bouveret y Fillmore (2008) reside, por consiguiente, no tanto en el particular del estudio, sino en la metodología planteada. Los autores subrayan que ambos modelos permiten analizar una pieza léxica en su contexto de uso: las 'funciones léxicas', por su capacidad de generar correlatos inmediatos de colocación, y los 'marcos', porque habilitan un entorno ontológico que permite comprender una unidad léxica concreta. Véanse los siguientes ejemplos para ilustrar esto:

(2) Funciones léxicas (FL) - en mayúsculas -

i. CAUSINCEPFACT $_{0}($ arma $)=$ disparar

ii. PREPARFACT $_{0}($ radiador $)=$ reglar

iii. LABREAL $_{12}($ ordenador $)=$ escribir $\{$ con/en $\}$

(3) Marcos semánticos - en mayúsculas -
i. $($ arma $\subset($ WEAPON $>$ SHOOTING) $) \rightarrow$ disparar
ii. (radiador $\subset$ ADJUSTING) $\rightarrow$ reglar
iii. (ordenador $\subset$ TEXT CREATION) $\rightarrow$ escribir $\{$ con/en $\}$

Las FL y los marcos semánticos son complementarios, hasta el punto de que, tal como

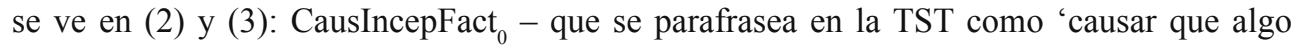
empiece a funcionar' - corresponde al marco WEAPON ${ }^{2}>\mathrm{SHOOTING}^{3}$ en la SM; la FL PreparFact $_{0}$ - 'preparar algo para que funcione' en la TST - corresponde al marco ADJUS$\mathrm{TING}^{4}$ en la SM; y $\mathrm{LABREAL}_{12}$ - FL conocida por constituir el uso de la entidad asociada a su tercer actante: instrumento o lugar - se ajusta al marco TEXT CREATION ${ }^{5}$. De esta forma, la TST con sus funciones léxicas proporciona un aparato formal de análisis léxico, en tanto que la SM ofrece un acercamiento más holístico e integrador del lenguaje. El empleo de estas herramientas guarda un importante potencial predictivo, tal y como puede confirmarse con la siguiente nómina de casos: i.) disparar puede ser base de pistola, fusil, escopeta, etc.; ii.) reglar de calentador, estufa, caldera, contador, etc.; iii.) escribir (con/ en\} de móvil, tableta, portátil, máquina de escribir, etc. Esta posibilidad, manifestada con los ejemplos de (2) y (3), constituye una de las principales prestaciones teóricas de la unión de las herramientas de ambos modelos.

La identificación colocacional a la que frecuentemente se hará referencia es entendida en este trabajo como la 'identificación de los patrones de la colocación'. Una de las incógnitas por esclarecer en la lexicología moderna es cómo puede sistematizarse una facultad como es la de consignar adyacentemente una pieza léxica a otra.

\footnotetext{
2 Arma.

3 Disparar.

4 Ajustar.

5 Creación de soporte textual.
} 
El aprendizaje de estos patrones, si bien es plenamente natural para un hablante - una circunstancia fundamentada en nociones como la institucionalización de estas combinaciones -, no lo es en absoluto para una realidad informática. Por todo ello, es una descripción de estas 'magnéticas' relaciones léxicas lo que se pretende con el presente trabajo. Un proceso genuinamente espontáneo pero que precisa de una formalización detallada.

Como hemos adelantado en 1.1, aquí hemos trabajado con el concepto de colocación de Mel'čuk (2006) en el que muchas colocaciones coinciden con lo que se ha etiquetado en otros enfoques como fenómenos de combinatoria (Bosque 2004). Un tipo de proceder que puede constatarse, por ejemplo, en la Teoría Sentido-Texto, fundamento teórico del trabajo, en el proyecto DiRetEs (FFI-2017-83293), influyente en la creación del corpus y la hipótesis inicial, o en interfaces digitales de búsqueda masiva de datos léxicos, como SketchEngine ( $<$ https://www.sketchengine.eu/ $>$ ).

Con todo, es evidente que una estructura de estas características tan generales es inabordable por cuanto sale a la luz un repertorio de datos inmanejable por su volumen. A este respecto, se ha optado por restringir el objeto hacia dos perspectivas que proporcionasen un significativo corpus de datos.

El corpus propuesto se ha ajustado, tanto en forma como en contenido. Dentro de las numerosas disposiciones en que se materializa la colocación, se ha optado por un análisis de aquellas estructuras de esquema verbonominal; esto es, aquellas combinaciones léxicas binarias que presentan una pieza nominal en actuación con una verbal. Resulta pertinente comentar que no se prestarán como relevantes en la clasificación las distintas configuraciones sintácticas que puedan darse entre estas dos categorías gramaticales. De este modo, se procederá a la identificación de todos los patrones colocacionales que caigan bajo el esquema verbonominal: bien cuando la pieza nominal se ejerza como sujeto (El altavoz funciona), bien como objeto directo (Encender el móvil) o bien como un complemento circunstancial (Escribir $\{c o n / e n\}$ el ordenador).

Una vez realizada esta primera selección de los datos, tomadas de las mencionadas fuentes, se procedió a realizar una delimitación temática del inventario. Dada la universalidad de las herramientas con que se trabajará, así como la necesidad de contar con un espectro de colocaciones lo suficientemente amplio, se optó finalmente por trabajar con un inventario de colocaciones de un ámbito suficientemente neutro y reconocible para los objetivos de identificación marcados. Entendiendo por aparato cualquier dispositivo provisto de un aparejo técnico mínimo y de creación humana, se recolectaron aquellas piezas léxicas que, desde las más rudimentarias (brújula, campana, etc.) hasta las más contemporáneas en su complejidad (ordenador, lavadora, cajero, coche, etc.), manifiestan empleos colocacionales representativos.

\subsection{Hipótesis de trabajo}

Identificar patrones colocacionales constituye una tarea ciertamente compleja por el volumen de datos con los que se opera, así como por la precisión que se debe tomar a la hora de realizar las anotaciones; la TST, en este sentido, proporciona una serie de herramientas con las que poder encarar este objeto de investigación. Tal es así que el mencionado proyecto DiRetEs (Barrios 2020), al cual se ha podido tener acceso, opera siguiendo la siguiente aplicación: 


\begin{tabular}{|l|c|c|}
\hline \multicolumn{1}{|c|}{ Función léxica } & Argumento & Valor \\
\hline Real $_{\perp}$ & Ordenador & Usar \\
\hline IncepReal $_{\perp}$ & Televisión & Encender \\
\hline LiquFact $_{0}$ & Motor & Apagar \\
\hline
\end{tabular}

Tabla 1: Modelo de aplicación

El empleo de estas herramientas, aunque luego se desarrolle con propiedad, retoma por completo el concepto de función como nexo entre un elemento que entra y otro que

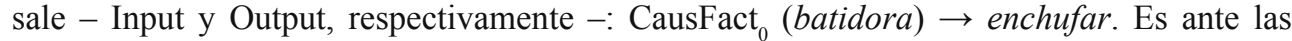
estrictas selecciones léxicas cuando este tipo de proceder es realmente rentable. Hay un notable número de compuestos en español que mantienen coexistencias de tal rigurosidad y singularidad que, con la mediación de estas funciones, quedan ilustradas nítidamente: Magn $($ comida $) \rightarrow$ opípara, Magn(esfuerzo) $\rightarrow$ hercúleo, etc. Por tanto, es esta precisión definitoria la principal ganancia que se obtiene con el manejo de la Teoría Sentido-Texto, especialmente de las funciones léxicas.

Por otro lado, las construcciones verbonominales que en este trabajo se abordan constan de un verbo de 'gobierno' conectado con un nombre dependiente. Es este elemento nominal lo que, bajo la SM, codifica un marco semántico determinado que contribuye al significado completo del conjunto.

La Semántica de Marcos, al igual que FrameNet, mantienen unos horizontes de investigación bastante más amplios que el estudio de las colocaciones. Para la identificación y uniformidad que aquí se pretende, el procedimiento es conciso y claro, habida cuenta de la complejidad conceptual que porta el marco intrínsecamente. El nombre dependiente - en la TST, argumento -, encargado de inducir la colocación, es el responsable de evocar aquel marco que lleva codificado y que, en última instancia, es el que despliega el significado del conjunto. El marco, bajo esta premisa, impone una serie de patrones colocacionales.

A título de ejemplo, cañón y rifle caen bajo el marco SHOOTING ARTIFACT ('artefacto de disparo'), que faculta, entre otras, algunas construcciones verbonominales con disparar: disparar el cañón; disparar el rifle, etc. Dos estructuras que se adscriben también a otros marcos, más estándares, como puede ser WEAPON ('arma'), donde coexisten con armas blancas; o más específicos, como puede ser BEARING ARMS ('armas de llevar consigo'). Una serie jerárquica de esferas conceptuales con las que se pueden trazar relevantes predicciones como la siguiente: disparar \{el cañón / \# el puñal\} o portar $\{$ el puñal / el rifle / \# el cañón $\}$. Unos contextos, que en muchos casos figuran jerarquizados, pero que, en su inmensa mayoría, precisan de la distinción explícita de las fronteras entre estos.

Sobre el tipo de metodología que se describe en la sección 2.3.1, ilustrada en la Tabla 1, en el corpus advertimos como una importante deficiencia las siguientes situaciones:

\begin{tabular}{|l|l|l|}
\hline \multicolumn{1}{|c|}{ Función léxica } & \multicolumn{1}{|c|}{ Argumento } & \multicolumn{1}{c|}{ Valor } \\
\hline \multirow{3}{*}{ Fact $_{0}$} & Bomba & Funcionar \\
\cline { 2 - 3 } & Bomba & Saltar \\
\cline { 2 - 3 } & Bomba & Explotar \\
\hline \multirow{2}{*}{ CausFunc $_{0}$} & Coche & Fabricar, Diseñar \\
\cline { 2 - 3 } & Coche & Publicar, Anunciar \\
\hline
\end{tabular}




\begin{tabular}{|c|l|l|}
\hline Función léxica & \multicolumn{1}{|c|}{ Argumento } & \multicolumn{1}{c|}{ Valor } \\
\hline \multirow{3}{*}{ LiquFact $_{0}$} & Ordenador & Estropear \\
\cline { 2 - 3 } & Ordenador & Suspender \\
\cline { 2 - 3 } & Ordenador & Apagar \\
\hline
\end{tabular}

Tabla 2: Ejemplos de deficiencias

Siendo evidente la productividad de las funciones léxicas, tal como muestra la Tabla 2, se ha advertido cierta sobregeneración por cuanto bajo una función con un mismo argumento pueden albergarse valores muy distintos. En esta línea, hemos decidido por considerar mejorable el tipo de formalización realizada en la Tabla 2.

Es en este punto donde se sostiene la implementación de un aparato semántico con la suficiente amplitud como para poder efectuar una sistematización mucho más exhaustiva de este conocimiento. La aplicación de los marcos permite, por un lado, profundizar con exactitud en cada patrón colocacional, y, por otro lado, abre un espectro de colocaciones en el que difícilmente podría haberse reparado. Un marco, en pocas palabras, proporciona un entorno ontológico sobre el que pueden motivarse distintas formas lingüísticas, de las que, asimismo, podrá derivarse un relevante etiquetado - o categorización léxica -. Es principalmente por esta eventualidad por la que se ha decidido poner en práctica la complementación de ambos modelos.

La siguiente tabla, sobre la sistematización de los datos presentados en la Tabla 2, per-

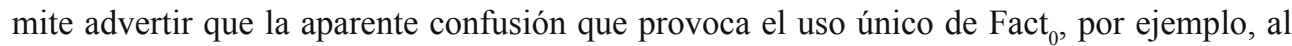
cubrir las colocaciones asociadas a bomba, sería aclarada con ayuda de los marcos semánticos. Una herramienta que, como puede observarse, abre unas posibilidades de granularidad en el análisis muy significativas, al margen del cometido discriminatorio que parece portar. A continuación, en la Tabla 3, se muestra lo que se pretende:

\begin{tabular}{|c|c|c|c|c|}
\hline $\begin{array}{l}\text { Función } \\
\text { Léxica } \\
\end{array}$ & \begin{tabular}{|l} 
Etiqueta \\
Semántica \\
\end{tabular} & $\begin{array}{l}\text { Marco } \\
\text { (en inglés) }^{6}\end{array}$ & $\begin{array}{l}\text { Base } \\
\text { verbal }\end{array}$ & Colocativo nominal \\
\hline \multirow[t]{3}{*}{ Fact $_{0}$} & APARATO & Tool_purpose & Funcionar & $\begin{array}{l}\text { Cualquier entidad contenida bajo APA- } \\
\text { RATO: móvil, estetoscopio, bomba, } \\
\text { regleta, coche, etc. }\end{array}$ \\
\hline & $\begin{array}{l}\text { APARATO } \\
\text { AUTÓNOMO }\end{array}$ & Getting_triggered & Saltar & $\begin{array}{l}\text { Cualquier entidad contenida bajo } \\
\text { APARATO AUTÓNOMO: alarma, } \\
\text { despertador, chivato, diferencial, } \\
\text { bomba, etc. }\end{array}$ \\
\hline & $\begin{array}{l}\text { APARATO } \\
\text { EXPLOSIVO }\end{array}$ & Explosion & $\begin{array}{l}\text { Explotar } \\
\text { Estallar }\end{array}$ & $\begin{array}{l}\text { Cualquier entidad contenida bajo APA- } \\
\text { RATO EXPLOSIVO: bomba, granada, } \\
\text { proyectil, mina, etc. }\end{array}$ \\
\hline
\end{tabular}

Tabla 3: Propuesta de formalización de un patrón colocacional ${ }^{6}$

Este ejemplo, sobre la sistematización de la Tabla 3, permite advertir que la aparente confusión que provoca el uso único de Fact, por ejemplo, al cubrir las colocaciones asociadas a bomba, sería aclarada mediante los marcos semánticos, una herramienta que, como puede constatarse, abre unas posibilidades de granularidad en el análisis muy significativas.

6 Marcos transcritos directamente desde FrameNet. 


\section{REVISIÓN DE LOS MODELOS TEÓRICOS}

\subsection{Semántica de Marcos}

\subsubsection{Marco como herramienta ontológica}

El primer fundamento del estudio se corresponde con la Semántica de Marcos. Un programa de semántica empírica, y ciertamente afín a un estudio etnográfico del lenguaje, que procede de los trabajos de Charles J. Fillmore (1976, 1982, 1985), quien fue el responsable de elaborar tanto las propuestas seminales del programa como aquellos trabajos posteriores que desembocarían en ambiciosos proyectos lexicológicos, entre los que se encuentra FrameNet (http://framenet.icsi.berkeley.edu) como más reconocido valedor. Este modelo está centrado en un concepto clave: el marco. Según la definición de Barcelona (2015: 147): "El término marco designa un modelo arraigado y rico en conocimientos, que opera recurrentemente sobre un área de experiencia bien delineada". El marco, de esta forma, se describe como una entidad continua que caracteriza una situación o escena determinada de forma muy esquematizada. Un caso de marco sería el siguiente:

(4) Marco TRANSACCIÓN

- 'el comprador' $\rightarrow$ comprar.

- 'el vendedor' $\rightarrow$ vender.

- 'el precio' $\rightarrow$ pagar.

En (4), el marco TRANSACCIÓN constituye un entorno con unas particularidades ontológicas con las que poder perspectivizar un evento de tres maneras distintas, cada manera aflorando un participante concreto: 'el comprador' en caso de comprar, 'el vendedor' en caso de vender y 'el precio' en caso de pagar.

La tesis que se ha planteado es sumamente sugerente en el ámbito de la semántica léxica en que se desarrolla. La palabra, entendida como lema, véase una unidad léxica dotada de autonomía, representaría, al margen del significado que lleva consigo, una categorización de la experiencia humana en aplicación principalmente de la T. ${ }^{a}$ de los prototipos (Rosch 1978). Por consiguiente, toda pieza léxica habría de analizarse sobre una situación motivante, un fondo de conocimientos y experiencias que avalen su empleo, así como el reconocimiento que los hablantes hacen de ella. Un ejemplo de esto sería el término desayuno, el cual evoca un marco fácilmente reconocible por su carácter convencional (primera comida del día, alimentos habituales que lo constituyen, realización comprendida en una horquilla horaria determinada, etc.); y otro caso ilustrativo podría ser la palabra elasticidad, que, en caso de emplearse con su sentido económico, necesita que el interlocutor le asigne un marco concreto para lograr la interpretación que se desea.

Dicha perspectiva de estudio llevaría a comprender que el comportamiento que ciertas palabras llevaban impreso en su categoría léxica permite entender una gramática mucho más amplia. El marco, de esta forma, se describiría como una entidad continua que, abstracta en su apariencia formal, caracteriza una situación o escena determinada de forma muy esquematizada. 


\subsubsection{FrameNet}

El programa informático FrameNet $<$ http://framenet.icsi.berkeley.edu $>$ constituye la principal herramienta sobre la que se sustenta la labor de reconocimiento, compendio y análisis de los diferentes marcos semánticos. Una interfaz de búsqueda léxica sobre la que se sustentará el posterior trabajo con datos.

Este recurso recoge el ideario completo de la Semántica de Marcos, por lo que constituye una prolongación de todos los conceptos tratados previamente; no obstante, dada la relevancia que adquirirá el portal a lo largo de la presentación y la discusión de los datos, resulta fundamental realizar una mínima descripción de FrameNet, así como de las facilidades que permite.

En líneas generales, FrameNet es un vasto proyecto lexicológico anglófono basado en la anotación manual - por colaboradores del proyecto - de un número inconmensurable de ejemplos léxicos con sus marcos correspondientes. Esta anotación elabora una serie de correspondencias a las que puede llegarse ya sea por las piezas léxicas integrantes o por los marcos semánticos. A nuestro juicio, es esta segunda, la clasificación relativa a los marcos, la que mayor amplitud goza, aunque hemos advertido importantes ausencias en el índice de unidades léxicas - p. ej. computer -.

Según la web, se estima una anotación de 200000 enunciados enlazados con un número cercano a los 1200 marcos. Una labor que, habiendo sido llevada a cabo por el Instituto de Ciencia Computacional de Berkeley desde el año 1997, atribuye a este programa una afinidad directa al inglés. Pese a ello, hemos decido optar por FrameNet dadas dos razones de peso:

- El carácter ontológico de la Semántica de Marcos permite que la inmensa mayoría de los marcos planteados y especificados sean plenamente extrapolables al español; una condición que ha puesto de relieve el carácter universal de esta línea de investigación.

- Por otro lado, el manejo de un corpus de ejemplos circunscrito al manejo de aparatos técnicos ha supuesto, en relación con el carácter universal mencionado antes, una similitud casi idéntica entre lenguas: un ordenador es un 'ordenador' en cualquier lengua, al igual que un microondas es un 'microondas'. No se advierte apenas ninguna interferencia cultural o idiomática entre el inglés y el español.

Comprendida la pertinencia de FrameNet para nuestro trabajo, se aportará a continuación una breve ilustración del programa. FrameNet presenta en su pantalla de inicio una larga serie de recursos, además de la presentación del modelo sobre el que se instaura. La labor que aquí se ha desarrollado parte del acceso en "Frame Index", como puede observarse en la figura 1. Una vez se ha ingresado en esta sección, podrá contemplarse un extenso listado de marcos del que, a pesar de su anotación manual en lengua inglesa, haremos uso en la labor de identificación de patrones colocacionales. 


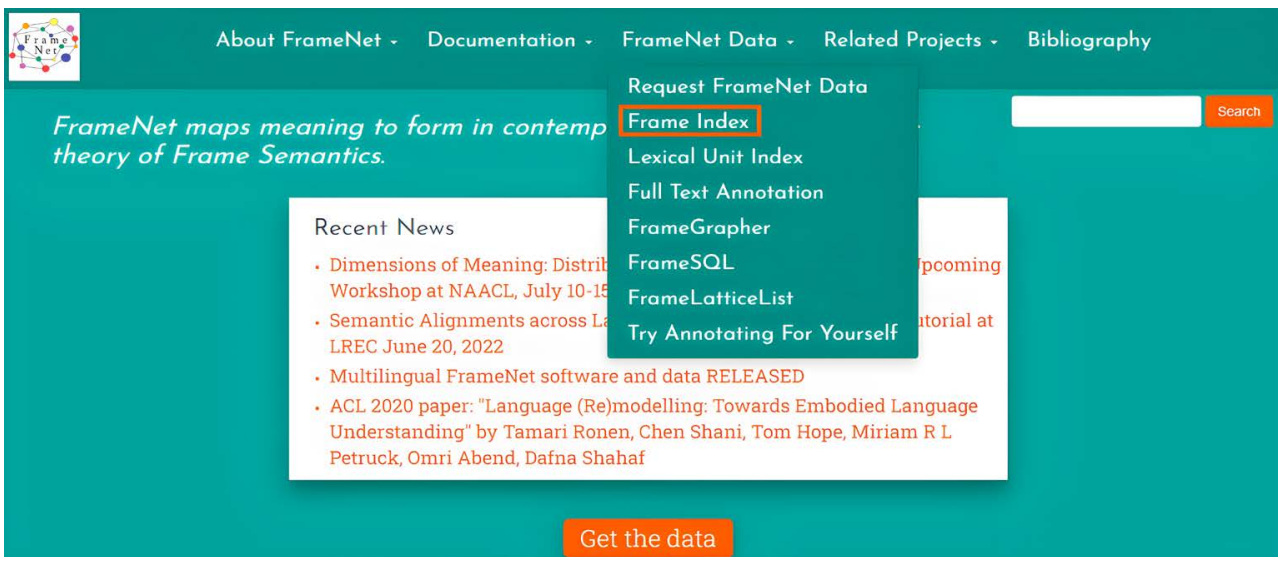

Fig. 1: Pantalla de inicio

Cada entrada hacia un marco despliega la consiguiente descripción, una caracterización del marco en la que se especifica con detalle el trasfondo ontológico básico a partir de cuatro ordenaciones. Tomando el marco SCRUTINY, generador de patrones con enfocar, centrar o apuntar $^{7}$, este se estructura en su definición de la siguiente manera:

\section{- Definición}

Como introducción al entorno ontológico, se expone una definición en la que se procuran condensar todos los ingredientes constitutivos del marco. Véase la figura 2:

\section{Scrutiny}

\section{Definition:}

This frame concerns a Cognizer (a person or other intelligent being) paying close attention to something, the Ground, in order to discover and note its salient characteristics. The Coonizer may be interested in a particular characteristic or entity, the Phenomenon that belongs to the Cround or is contained in the Groun (or to ensure that such a property of entity is not present). Some words in this frame allow alternate expressions of the Groundand the Phenomenon: We SEARCHED the yar for my contact lens.

Fig. 2: Ejemplo de definición

\section{- Elementos de marco}

Los elementos de marco (Frame Elements), entendidos como los participantes del evento, están descritos a continuación. Véase en la figura 3 las descripciones semántico-sintácticas de Cognizador, Fondo, Instrumento o Medio:

7 V.g.: Enfoca bien el escáner; Esta cámara centra automáticamente el objetivo; Apunta el telescopio hacia la constelación; Los prismáticos del coche no enfocan. 


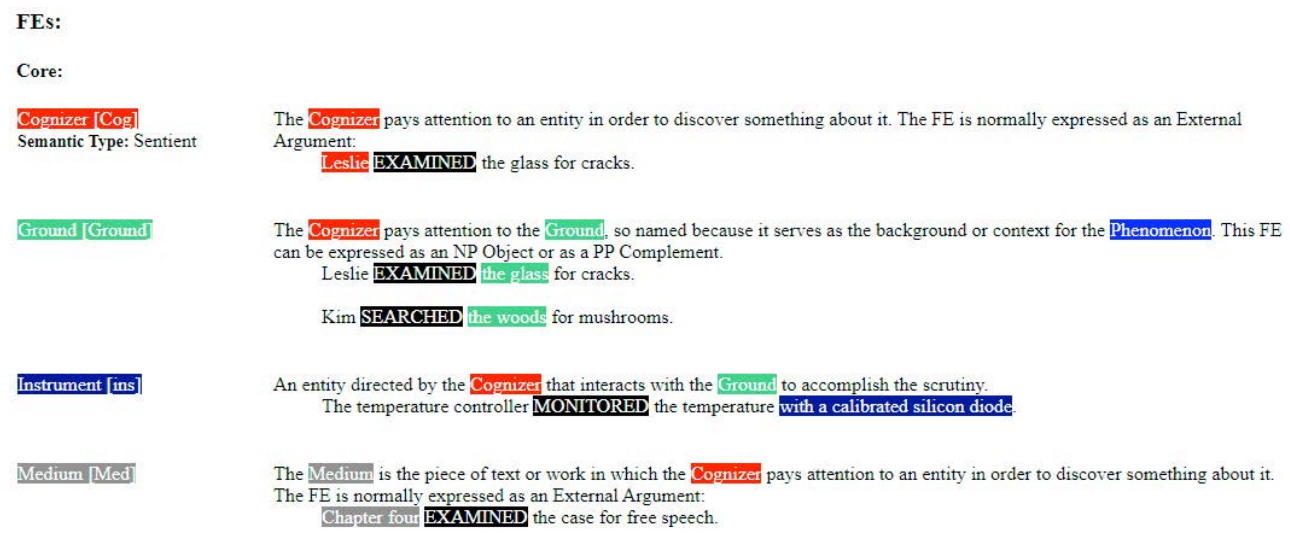

Fig. 3: Ejemplo de elementos del marco SCRUTINY

- Relaciones entre marcos

La entrada del marco provee en última instancia un significativo desglose de las relaciones que el marco descrito puede guardar con otros marcos (relaciones de herencia, de perspectivización de un evento, de jerarquía ontológica, de precedencia, etc.). Una labor de vinculación que se presta muy valiosa a la hora de establecer importantes generalizaciones, como que una unidad léxica coexista en una larga serie de marcos enlazados por relaciones de herencia. Véase la figura 4:

\section{Frame-frame Relations:}

Inherits from:

Is Inherited by: Reading_perception, Research, Scrutinizing_for, Verification

Perspective on:

Is Perspectivized in:

Uses: Becoming_aware, Perception_active

Is Used by: Experimentation

Subframe of:

Has Subframe(s):

Precedes:

Is Preceded by:

Is Inchoative of:

Is Causative of:

See also: Seeking

Fig. 4: ejemplo de listado de relaciones de marco a marco

\subsection{Teoría Sentido-Texto}

La Teoría Sentido-Texto (Mel’čuk 1995, 1996, 2015a, etc.) constituye, por otro lado, un modelo lexicológico singular por las premisas teóricas que propone, así como por la metodología concreta con que opera. La contribución más relevante de la TST ha sido aportar un amplio repertorio de herramientas formales, conocidas con el nombre de funciones léxicas (FL), que permiten describir rigurosamente las relaciones semánticas y léxicas. 
De acuerdo con Mel'čuk (1996: 38), la función léxica se define como “el conjunto de herramientas formales diseñadas para la descripción, de una manera compacta y sistemática, de todas las genuinas relaciones léxicas que se obtienen con piezas léxicas de cualquier lengua" (traducción propia).

Véanse los siguientes ejemplos de (5):

(5) i. esp. Magn (recomendar $)=$ encarecidamente, vivamente, firmemente, enérgicamente ii. ${ }_{\text {fr. }}$ Magn $($ recommender $)=$ fortement, vivement, vigoureusement, instamment iii. ${ }_{\text {ing. }}$ Magn $($ recomend $)=$ highly, strongly, heartily

Como se observa en los ejemplos de (5), la utilidad de las FL es habilitar una sistematización de este fenómeno de selección léxica. La noción de función, recogida desde su sentido matemático tradicional, implica, en consecuencia, tres componentes: una función (Magn: 'en alto grado'), aplicada sobre un argumento - o palabra clave - (recomendar), y un valor (encarecidamente), que especifica el resultado de la operación analítica.

Las FL operan en dos ejes claros: paradigmático y sintagmático. En nuestro caso, nos interesamos particularmente por aquellas sintagmáticas; aquellas formuladas para agrupar dos unidades léxicas de una manera en la que la coocurrencia sea restringida y no libre. En otras palabras, suele haber un patrón de coocurrencia que no debe violarse, puesto que rige con qué unidad léxica puede combinarse otra unidad léxica.

Asimismo, estas herramientas son predicados por cuanto son capaces de desarrollar una abreviada estructura temática. Los participantes, llamados con el nombre de actantes en la terminología de la TST, son a todas luces una serie de variables argumentales, como puede verse en (6):

(6) 0: Participante vacío - Func $_{0}($ dia $)=$ transcurrir $;$ FinFact $_{0}($ ordenador $)=$ congelarse .

I: Primer participante: $\underline{X}-\operatorname{Real}_{1}($ televisión $)=$ ver; $\operatorname{Fact}_{1}($ chaqueta $)=$ abrigar.

II: Segundo participante: $\mathrm{X}$ e $\underline{\mathrm{Y}}-\mathrm{Func}_{2}($ castigo $)=$ aplicar a $X$ un .

A continuación, veamos la serie de ejemplos de (7) para introducir algunas de las FL que hemos empleado en la formalización de nuestro corpus:

(7) Real $_{0 / i}$ : Correspondida con el uso humano de la entidad.

V.g.: Real $($ guitarra $)=$ tocar $; \operatorname{Real}_{1}($ móvil $)=$ usar .

Fact $_{\text {0/i }}$ : Correspondida con la utilidad de la entidad (¿Para qué ha sido creada?).

V.g.: Fact $_{0}($ coche $)=$ funcionar; Fact $_{1}($ espejo $)=$ reflejar (algo).

Func: 'existir de alguna forma'.

V.g.: Func $_{0}($ dia $)=$ transcurrir; Func $_{1}($ sol $)=$ quemar (algo/alguien $)$.

Estas FL, asimismo, presentan la capacidad de poder ensamblarse con otras para la expresión de significados más amplios, como puede verse en (8):

(8) Incep: Encargada de dotar un valor incoativo o ingresivo.

V.g.: IncepOper ${ }_{1}($ felicidad $)=$ encontrar; IncepReal $_{1}($ coche $)=$ abrir .

Caus: Causar que V (donde V es un verbo, como en causar que algo exista o causar que algo funcione).

V.g.: CausFunc $_{0}($ edificio $)=$ construir $;$ CausIncepFact $_{0}($ televisión $)=$ encender . 
Del mismo modo, algunos modificadores adjetivo-adverbiales que puedan ser necesarios en los datos ya expuestos o que se expondrán a continuación, son los que se muestran en (9):

(9) Magn: 'en un alto grado'; Anti: relación básica de antonimia; Excess: 'de manera desmesurada'; Prepar: 'preparar algo para un fin'.

V.g.: AntiMagn $($ pelo $)=$ ralo; AntiReal $_{1}($ reloj $)=$ robar; $\operatorname{PreparFact~}_{0}($ gafas $)=$ limpiar $_{\text {; }}$ Excess $($ corazón $)=$ acelerarse $;$ PreparFact $_{0}($ ensalada $)=$ aliñar .

Por otra parte, el Principio de Herencia Léxica, enunciado por vez primera en Mel'čuk y Wanner (1996), resulta el fundamento teórico-metodológico de nuestro estudio. Este consiste en rastrear aquellos rasgos semánticos que, compartidos entre un número considerable de unidades léxicas, explica, por ejemplo, que un patrón colocacional se repita en una variedad de casos. Véanse los ejemplos de (10):

$$
\begin{aligned}
& \text { i. } \left.\text { CausFact }_{0} \text { (televisor }\right)=\text { enchufar. } \\
& \text { ii. CausFact } \left.\text { (batidora })=\text { enchufar. }_{\text {(iii. CausFact }} \text { (ventilador }\right)=\text { enchufar } \text {. }
\end{aligned}
$$

Las unidades léxicas que operan en calidad de argumento en los ejemplos de (10) se archivan bajo un mismo campo semántico. La unificación extraída de CausFact, con enchufar como valor resultante, opera con los argumentos existentes en la categoría de APARATO CON CABLE, un lexema genérico y, simultáneamente, un designador de un campo concreto de sustantivos. Siguiendo a Polguère (2003), esta categoría taxonómica adquiere la denominación de etiqueta semántica.

\section{ANÁLISIS DE LOS DATOS}

A continuación, expondremos los casos más relevantes de la propuesta de sistematización que hemos elaborado. El extenso listado de sistematizaciones que se ha realizado en el trabajo ha permitido que apreciemos el marco como un elemento sumamente versátil a la hora de proporcionar soluciones de análisis.

Conviene advertir que todos los datos son de creación propia. Los marcos aducidos han sido extraídos de FrameNet, con la salvedad de que se realiza una traducción propia de cada descripción del marco. Por último, las formalizaciones con FL han sido ideadas también por cuenta propia, con el auxilio de muchos de los trabajos citados previamente.

\subsection{Marco como herramienta discriminatoria}

En virtud de lo advertido en la hipótesis inicial, se ha confirmado la capacidad del marco para distinguir entre manifestaciones distintas de una misma FL una facultad que ha sido apreciable con claridad en aquellas FL más frecuentes.

\subsubsection{Real}

Se ha podido constatar cómo un valor concreto de una FL puede materializarse en muy distintas realizaciones lingüísticas según el sentido ontológico que se haya adoptado. 
Para la primera tabla y las siguientes que se expongan, estas disposiciones constan de una subtabla en la que se describe el patrón con las herramientas formales - la TST propone Real $_{1}$ (batidora) $=$ usar y la SM, el marco Using -, para posteriormente continuar con una segunda subtabla, en la que se detalla lingüísticamente el patrón colocacional, mediante las bases verbales y los colocativos nominales que correspondan.

En la Tabla 6 podemos ver uno de los patrones colocacionales más generales y sobre el que caerían todos los aparatos que se han concebido en nuestro corpus, aquellos bajo la etiqueta de APARATO:

\begin{tabular}{|l|l|l|l|}
\hline Función Léxica & $\begin{array}{l}\text { Etiqueta } \\
\text { Semántica }\end{array}$ & Marco & Descripción resumida del marco \\
\hline Real $_{1}$ & APARATO & Using & $\begin{array}{l}\text { Un Agente manipula una Entidad con el objeto de } \\
\text { lograr un Propósito. }\end{array}$ \\
\hline
\end{tabular}

\begin{tabular}{|c|c|c|}
\hline Base verbal & $\begin{array}{l}\text { Colocativo nominal (como } \\
\text { OD) }\end{array}$ & Ejemplos \\
\hline $\begin{array}{l}\text { Usar; Utilizar; } \\
\text { Manejar; Emplear. }\end{array}$ & $\begin{array}{l}\text { Cualquier entidad conteni- } \\
\text { da bajo APARATO: batido- } \\
\text { ra, escáner, coche, pistola, } \\
\text { bisturí, móvil, etc. }\end{array}$ & $\begin{array}{l}\text { Juan usó la batidora para la tarta; Es nece- } \\
\text { sario utilizar el escáner; No manejes aún el } \\
\text { coche; Ya puedes emplear la pistola; Usa el } \\
\text { bisturí; Los asaltantes emplearon un arma de } \\
\text { fuego; No puedes utilizar el móvil durante el } \\
\text { examen. }\end{array}$ \\
\hline
\end{tabular}

Tabla 6: 1.er patrón colocacional de Real $_{1}$

En la Tabla 7, Real1 sería una función léxica ya discriminada con la intercesión del marco Operate_vehicle:

\begin{tabular}{|l|l|l|l|}
\hline Función Léxica & Etiqueta Semántica & Marco & Descripción resumida del marco \\
\hline Real $_{1}$ & $\begin{array}{l}\text { APARATO DE } \\
\text { TRANSPORTE }\end{array}$ & Operate_vehicle & $\begin{array}{l}\text { Un Agente controla un Vehículo en } \\
\text { movimiento. }\end{array}$ \\
\hline
\end{tabular}

\begin{tabular}{|l|l|l|}
\hline Base verbal & Colocativo nominal (como OD) & Ejemplos \\
\hline $\begin{array}{l}\text { Llevar; Manejar; } ; \\
\text { Controlar; Condu- } \\
\text { cir; Pilotar. }\end{array}$ & $\begin{array}{l}\text { buajo APARAR entidad contenida } \\
\text { PORTE: coche, tren, barco, },\end{array}$ & $\begin{array}{l}\text { Lleva el coche un rato; El PER permite ma- } \\
\text { nejar barcos; } \text { No puedes conducir autobuses } \\
\text { avioneta, autobús, etc. } ; \text { Con qué soltura controla el tren!; } \\
\text { En un año sabrás pilotar una avioneta. }\end{array}$ \\
\hline
\end{tabular}

Tabla 7: $2^{\mathrm{o}}$ patrón colocacional de Real ${ }_{1}$

En esta segunda sistematización, la discriminación se realizaría tomando en consideración el marco de Explosion, inductor de determinadas bases verbales, como se aprecia en la Tabla 8:

\begin{tabular}{|l|l|l|l|}
\hline Función Léxica & Etiqueta Semántica & Marco & Descripción resumida del marco \\
\hline Real $_{1}$ & $\begin{array}{l}\text { APARATO } \\
\text { EXPLOSIVO }\end{array}$ & Explosion & $\begin{array}{l}\text { Debido a una reacción química o nuclear, una } \\
\text { Entidad explota provocando un Daño signifi- } \\
\text { cativo en su área circundante. }\end{array}$ \\
\hline
\end{tabular}




\begin{tabular}{|l|l|l|}
\hline Base verbal & Colocativo nominal (como OD) & Ejemplos \\
\hline Detonar; & $\begin{array}{l}\text { Cualquier entidad contenida bajo APA- } \\
\text { Estallar; Activar. } \\
\text { RATO EXPLOSIVO: bomba, granada, } \\
\text { proyectil, mina, etc. }\end{array}$ & $\begin{array}{l}\text { El artillero detonó las bombas cuida- } \\
\text { dosamente; Con la anilla no podrás } \\
\text { estallar la granada; Activaron la mina } \\
\text { al paso de las tropas enemigas. }\end{array}$ \\
\hline
\end{tabular}

Tabla 8: 3.er patrón colocacional de Real $_{1}$

La realidad a la que se quiere llegar con estas descripciones es el hecho de que, evidentemente, todos los aparatos se usan, pero no todos pueden conducirse o detonarse. Es necesario contar con un parámetro que discrimine el empleo que cada entidad requiera o admita.

\subsubsection{Fact $_{1}$}

En el caso de la función léxica Fact, el cometido discriminatorio de los marcos se hace más necesario que antes en la medida en que los distintos patrones de Fact $_{1}$ operan bajo una misma etiqueta semántica: APARATO INFORMÁTICO.

La Tabla 9 desarrollaría Fact $_{1}$ con respecto a Mining, que es la extracción de datos digitales con medios informáticos:

\begin{tabular}{|l|l|l|l|}
\hline $\begin{array}{l}\text { F u n c i ó n } \\
\text { Léxica }\end{array}$ & Etiqueta Semántica & Marco & Descripción resumida del marco \\
\hline Fact $_{1}$ & $\begin{array}{l}\text { APARATO } \\
\text { INFORMÁTICO }\end{array}$ & $\begin{array}{l}\text { Mining } \\
< \\
\text { Perception }\end{array}$ & $\begin{array}{l}\text { Una Entidad manejada por un Agente pre- } \\
\text { senta unas facultades apropiadas para la ex- } \\
\text { tracción y compilación de Información. }\end{array}$ \\
\hline
\end{tabular}

\begin{tabular}{|l|l|l|}
\hline Base verbal & Colocativo nominal (como sujeto) & Ejemplos \\
\hline Obtener; Recabar; $;$ & Cualquier entidad contenida bajo & El ordenador obtiene los resultados fis- \\
Compilar; Recopi- & APARATO INFORMÁTICO: orde- & cales muy rápido; El escáner compila \\
lar. & $\begin{array}{l}\text { nador, móvil, tableta, escáner, caje- } \\
\text { ro, etc. }\end{array}$ & $\begin{array}{l}\text { los documentos en un mismo PDF; La } \\
\text { tableta recopila los gastos del mes; Es- } \\
\text { pera a que el cajero recabe todas tus } \\
\text { credenciales. }\end{array}$ \\
\hline
\end{tabular}

Tabla 9: 1.er patrón colocacional de Fact $_{1}$

En cambio, Fact $_{1}$ con Becoming_aware permitiría la identificación clara de otra serie de patrones colocacionales para APARATOS INFORMÁTICOS, como se ve en la Tabla 10:

\begin{tabular}{|l|l|l|l|}
\hline Función Léxica & $\begin{array}{l}\text { Etiqueta } \\
\text { Semántica }\end{array}$ & Marco & Descripción resumida del marco \\
\hline Fact $_{1}$ & $\begin{array}{l}\text { APARATO } \\
\text { INFORMÁTICO }\end{array}$ & $\begin{array}{l}\text { Becoming_aware } \\
< \\
\text { Perception }\end{array}$ & $\begin{array}{l}\text { Una Entidad manejada por un Agente } \\
\text { presenta unas capacidades cognitivas } \\
\text { suficientes como para advertir, identificar } \\
\text { y rotular un Fenómeno concreto. }\end{array}$ \\
\hline
\end{tabular}




\begin{tabular}{|l|l|l|}
\hline Base verbal & Colocativo nominal (como sujeto) & Ejemplos \\
\hline $\begin{array}{l}\text { Detectar; Descubrir; } ; \text { Identificar; Comprobar. } \\
\text { Cualquier entidad contenida bajo }\end{array}$ & $\begin{array}{l}\text { La impresora detectó defectos de } \\
\text { denador, móvil, tableta, impresora, or }, \\
\text { forma; Deja que el ordenador iden- } \\
\text { tifique la web; El móvil comprueba } \\
\text { el stock automáticamente; El GPS } \\
\text { ha descubierto una ruta. }\end{array}$ \\
\hline
\end{tabular}

Tabla 10: $2^{\circ}$ patrón colocacional de Fact1

Y, por último, Fact $_{1}$, a propósito de Research, identifica con precisión un grupo de patrones colocacionales que difiere bastante de los anteriores presentados bajo la misma función léxica. La Tabla 11 lo ilustra:

\begin{tabular}{|l|l|l|l|}
\hline $\begin{array}{l}\text { Función } \\
\text { Léxica }\end{array}$ & Etiqueta Semántica & Marco & Descripción resumida del marco \\
\hline Fact & $\begin{array}{l}\text { APARATO } \\
\text { INFORMÁTICO }\end{array}$ & $\begin{array}{l}\text { Research } \\
< \\
\text { Perception }\end{array}$ & $\begin{array}{l}\text { Una Entidad manejada por un Agente presenta unas } \\
\text { capacidades cognitivas suficientes para el rastreo y } \\
\text { análisis de volúmenes de Datos. }\end{array}$ \\
\hline
\end{tabular}

\begin{tabular}{|c|c|c|}
\hline Base verbal & $\begin{array}{l}\text { Colocativo nominal (como } \\
\text { sujeto) }\end{array}$ & Ejemplos \\
\hline $\begin{array}{l}\text { Analizar; Examinar; } \\
\text { Rastrear; Explorar; } \\
\text { Buscar. }\end{array}$ & $\begin{array}{l}\text { Cualquier entidad conte- } \\
\text { nida bajo APARATO IN- } \\
\text { FORMÁTICO: ordenador, } \\
\text { móvil, tableta, impresora, } \\
\text { GPS, etc. }\end{array}$ & $\begin{array}{l}\text { ¿Mi móvil puede explorar PDF?; Un ordena- } \\
\text { dor rastrea información constantemente; El El } \\
\text { GPS aún está buscando las coordenadas; Las } \\
\text { impresoras examinan el documento; Un servi- } \\
\text { dor analiza diariamente los accesos web. }\end{array}$ \\
\hline
\end{tabular}

Tabla 11: 3.er patrón colocacional de Fact1

\subsection{Marco como herramienta etiquetadora}

La clasificación en etiquetas semánticas permite ilustrar de manera muy específica qué tipo de aparatos caen bajo un determinado patrón colocacional. En esta línea, los marcos han desarrollado la labor de ser capaces de inducir la creación de ciertos etiquetados semánticos.

\subsubsection{APARATO INFORMÁTICO < Perception}

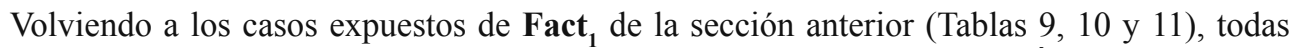
las sistematizaciones que caen bajo la etiqueta de APARATO INFORMÁTICO mantienen como origen de ser el marco Perception. Un entorno ontológico responsable de designar que la entidad en cuestión, aparato o no, presenta unas capacidades cognitivas suficientes para realizar determinadas labores: ya sea de extracción de datos, de identificación, o de rastreo y análisis de volúmenes de información. 


\subsubsection{APARATO DE INDICACIÓN INFORMATIVA < Information_display}

Un caso ilustrativo es el marco Information_display. Un marco semántico que ha permitido circunscribir aquellos aparatos destinados a la presentación de una información o, como su nombre bien indica, de INDICACIÓN INFORMATIVA. La Tabla 12 expone esta descripción:

\begin{tabular}{|l|l|l|l|}
\hline Función Léxica & $\begin{array}{l}\text { Etiqueta } \\
\text { Semántica }\end{array}$ & Marco & Descripción resumida del marco \\
\hline Fact $_{1}$ & $\begin{array}{l}\text { APARATO DE } \\
\text { INDICACIÓN } \\
\text { INFORMATIVA }\end{array}$ & Information_display & $\begin{array}{l}\text { Una Entidad física de creación hu- } \\
\text { mana es empleada para presentar una } \\
\text { Información determinada. }\end{array}$ \\
\hline
\end{tabular}

\begin{tabular}{|l|l|l|}
\hline Base verbal & Colocativo nominal (como sujeto) & Ejemplos \\
\hline Enseñar; Mostrar; Re- & Cualquier entidad contenida bajo & El ordenador refleja los resultados \\
flejar; Describir. & APARATO DE IND. INFORMATI- & del ejercicio; El móvil ya te enseña \\
& VA: ordenador, portátil, móvil, ta- & el camino; No ignores lo que mues- \\
& $\begin{array}{l}\text { bleta, televisión, monitor, pantalla, }, \\
\text { radion } \text { tas pantallas; } \text { La televisión des- } \\
\text { raibe la actualidad. }\end{array}$ \\
\hline
\end{tabular}

Tabla 12: Patrón colocacional de APARATOS DE INDICACIÓN INFORMATIVA

\subsubsection{APARATO DE ACTUACIÓN MESURABLE < Measurable_attributes}

Un último caso que cabe destacar para ilustrar el cometido etiquetador atribuido al marco es el de aquellos aparatos que tienden a funcionar de manera desmesurada en ciertos contextos. La simple adopción de la FL Excess, ciertamente 'ad-hoc', no parecía suficiente. Un patrón colocacional que, sin duda, queda registrado de forma más unívoca con el submarco Surpassing, que hereda de Measurable_attributes, el marco concreto que motiva la creación de la etiqueta APARATO DE ACTUACIÓN MESURABLE. Véase la Tabla 13:

\begin{tabular}{|l|l|l|l|}
\hline Función Léxica & $\begin{array}{l}\text { Etiqueta } \\
\text { Semántica }\end{array}$ & Marco & Descripción resumida del marco \\
\hline Excess & $\begin{array}{l}\text { APARATO DE } \\
\text { ACT. MESU- } \\
\text { RABLE }\end{array}$ & $\begin{array}{l}\text { Surpassing } \\
< \\
\text { Measurable_attributes }\end{array}$ & $\begin{array}{l}\text { Una Entidad física manejada por un } \\
\text { Agente despliega en su Actuación una } \\
\text { serie de Atributos que, ya sea cualitativa } \\
\text { como cuantitativamente, sobrepasan los } \\
\text { Parámetros habituales. }\end{array}$ \\
\hline
\end{tabular}

\begin{tabular}{|c|c|c|}
\hline Base verbal & Colocativo nominal (como OD) & Ejemplos \\
\hline $\begin{array}{l}\text { Sobrecalentar; Sobre- } \\
\text { cargar; Revolucionar; } \\
\text { Asfixiar. }\end{array}$ & $\begin{array}{l}\text { Cualquier entidad contenida bajo } \\
\text { APARATO DE ACT. MESURABLE: } \\
\text { coche, móvil, transformador, carga- } \\
\text { dor, fusibles, etc. }\end{array}$ & $\begin{array}{l}\text { ¿Que estás asfixiando el motor!; No } \\
\text { vas a sobrecalentar ningún fusible; } \\
\text { Mejor evita revolucionar el coche; } \\
\text { Sobrecargaste mucho el móvil. }\end{array}$ \\
\hline
\end{tabular}

Tabla 13: Patrón colocacional de APARATOS DE ACTUACIÓN MESURABLE 


\subsection{Marco como herramienta especificadora}

En última instancia, se ha señalado que, a través del marco, es posible desarrollar la posibilidad de concretar la forma en que un marco se manifiesta. En otras palabras, el marco, por medio de sus relaciones, de herencia, puede especificar con mayor detalle su naturaleza, así como la de los patrones colocacionales que identifica.

\subsubsection{AntiReal con \{Theft / Theft $<$ Money\}}

Un ejemplo esclarecedor se presenta con el marco Theft, que contemplaría el robo en su nivel más básico: la apropiación física de una entidad (robar, apoderarse de o llevarse un APARATO). No obstante, si este mismo marco es heredado por uno en que se realce un tema monetario, como es Money, el sentido del robo es distinto, puesto que se contempla la retirada violenta de un tema monetario contenido en una entidad y no la apropiación de una entidad en sí. En las Tablas 14 y 15 pueden verificarse estas dos situaciones:

\begin{tabular}{|l|l|l|l|}
\hline Función Léxica & $\begin{array}{l}\text { Etiqueta } \\
\text { Semántica }\end{array}$ & Marco & Descripción resumida del marco \\
\hline AntiReal $_{1}$ & APARATO & Theft & $\begin{array}{l}\text { Un Agente asume violentamente el Control físico de una } \\
\text { Entidad propiedad de un Tercero. }\end{array}$ \\
\hline
\end{tabular}

\begin{tabular}{|c|c|c|}
\hline Base verbal & Colocativo nominal (como OD) & Ejemplos \\
\hline $\begin{array}{l}\text { Robar; Apoderarse de; } \\
\text { Llevarse. }\end{array}$ & $\begin{array}{l}\text { Cualquier entidad contenida bajo } \\
\text { APARATO: coche, móvil, lavadora, } \\
\text { prismáticos, televisión, etc. }\end{array}$ & $\begin{array}{l}\text { Los ladrones se apoderaron de los } \\
\text { coches; Nos robaron hasta el co- } \\
\text { che; Se llevaron varios móviles. }\end{array}$ \\
\hline
\end{tabular}

Tabla 14: Patrón colocacional de AntiReal $_{1}$ para Theft

\begin{tabular}{|l|l|l|l|}
\hline Función Léxica & $\begin{array}{l}\text { Etiqueta Semán- } \\
\text { tica }\end{array}$ & Marco & Descripción resumida del marco(s) \\
\hline AntiReal $_{1}$ & $\begin{array}{l}\text { APARATO DE } \\
\text { GESTIÓN PE- }\end{array}$ & $\begin{array}{l}\text { Theft } \\
\text { CUNIARIA }\end{array}$ & $\begin{array}{l}\text { Una Entidad física, concebida como un medio de ges- } \\
\text { tión de efectivos monetarios, es involucrada en una } \\
\text { situación en la que un Agente retira violentamente un } \\
\text { Tema económico de una Fuente concreta. }\end{array}$ \\
\hline
\end{tabular}

\begin{tabular}{|c|c|c|}
\hline Base verbal & Colocativo nominal (como OD) & Ejemplos \\
\hline $\begin{array}{l}\text { Robar; Saquear; Desvali- } \\
\text { jar; Asaltar. }\end{array}$ & $\begin{array}{l}\text { Cualquier entidad contenida bajo } \\
\text { APARATO DE GESTIÓN PECU- } \\
\text { NIARIA: caja registradora, cajero, } \\
\text { caja acorazada, casillero, depósito, } \\
\text { etc. }\end{array}$ & $\begin{array}{l}\text { No quiero que me roben el ca- } \\
\text { sillero; Saquearon la caja aco- } \\
\text { razada; Ese cajero lo desvali- } \\
\text { jaron ayer; Los delincuentes no } \\
\text { asaltaron la caja registradora. }\end{array}$ \\
\hline
\end{tabular}

Tabla 15: Patrón colocacional de AntiReal $_{1}$ para Theft $<$ Money 


\subsubsection{PreparFact ${ }_{0}$ con Containers \{Container_focused_placing / Container_focused_ removing\}}

Un caso en el que es evidente la jerarquización de los marcos, así como las distintas realizaciones que un marco puede presentar, es el manifestado por los APARATOS CONTINENTES. De acuerdo con el submarco con que se construya habrá un patrón colocacional u otro. Por un lado, Container_focused_placing para llenar o colmar - esto es, la situación de un tema en un continente - y, por otro lado, Container_focused_removing para vaciar o purgar (la retirada de un tema de un continente). Las Tablas 16 y 17 ilustran estos dos patrones colocacionales, respectivamente:

\begin{tabular}{|l|l|l|l|}
\hline Función Léxica & $\begin{array}{l}\text { Etiqueta } \\
\text { Semántica }\end{array}$ & Marco & Descripción resumida del marco \\
\hline PreparFact & $\begin{array}{l}\text { A P A R A T O } \\
\text { CONTINENTE }\end{array}$ & $\begin{array}{l}\text { Container_focused_placing } \\
< \\
\text { Containers }\end{array}$ & $\begin{array}{l}\text { Una Entidad concebida prototípica- } \\
\text { mente para cumplir un Cometido de } \\
\text { contención, física o digital. En este } \\
\text { caso, un Agente o Causa se encarga } \\
\text { de situar un Tema en el Continente. }\end{array}$ \\
\hline
\end{tabular}

\begin{tabular}{|l|l|l|}
\hline Base verbal & Colocativo nominal (como OD) & Ejemplos \\
\hline Llenar; Colmar; & Cualquier entidad contenida bajo & No me llenes el maletero; Llenar el móvil \\
Inflar/Hinchar. & APARATO CONTINENTE: ordena- & nunca es recomendable; Hincha el balón; \\
& dor, maletero, depósito, balón, etc. & Hay que colmar el depósito antes de viajar. \\
\hline
\end{tabular}

Tabla 16: Patrón colocacional de PreparFact ${ }_{0}$ para Container_focused_placing < Containers

\begin{tabular}{|l|l|l|l|}
\hline Función Léxica & $\begin{array}{l}\text { Etiqueta } \\
\text { Semántica }\end{array}$ & Marco & Descripción resumida del marco \\
\hline PreparFact & $\begin{array}{l}\text { A P A R A T O } \\
\text { CONTINEN- }\end{array}$ & $\begin{array}{l}\text { Container_focused_removing } \\
\text { TE }\end{array}$ & $\begin{array}{l}\text { Una Entidad concebida prototí- } \\
\text { picamente para cumplir un Co- } \\
\text { metido de contención, física o } \\
\text { digital. En este caso, un Agente } \\
\text { o Causa se encarga de retirar un } \\
\text { Tema del Continente. }\end{array}$ \\
\hline
\end{tabular}

\begin{tabular}{|l|l|l|}
\hline Base verbal & Colocativo nominal (como OD) & Ejemplos \\
\hline Vaciar; Purgar. & Cualquier entidad contenida bajo & Purga todos los radiadores antes de \\
& APARATO CONTINENTE: ordena- - & abandonar; No vacíes aún el ordenador; \\
dor, disco duro, coche, depósito, cár- - & $\begin{array}{l}\text { Pidele a tu hermano que vacie el coche } \\
\text { de maletas; Hay que purgar el cárter de } \\
\text { teradiador, etc. }\end{array}$ & \\
\hline
\end{tabular}

Tabla 17: Patrón colocacional de PreparFact 0 para Container_focused_removing $<$ Containers

\section{CONCLUSIONES}

Como ya mencionamos en el apartado metodológico, el corpus seleccionado para la investigación cumplía las necesidades que en un primer momento se sostuvieron. La elección de un tipo de colocación concreto ha satisfecho completamente nuestras previsiones; tanto por 
la elección de un esquema verbonominal como por la temática relativa a los aparatos. Sobre este conjunto de datos se ha podido generar una más que numerosa relación de sistematizaciones, de la cual, por otro lado, se ha tenido que desestimar para esta publicación un gran número de casos por motivos de espacio.

La hipótesis inicial expuesta daba a conocer una situación de descripción que advertimos como posiblemente perfectible. La identificación de patrones colocacionales ha resultado la labor que, al margen de la pertinente descripción lingüística de la colocación, se ha perseguido a lo largo del estudio. La TST, y en particular las funciones léxicas, constituyen una herramienta excepcional de identificación del correlato colocacional de una pieza léxica. Sin embargo, este proceder ha desvelado una serie de posibles mejoras, como la descripción que se hace de ciertas colocaciones - unificándolas, pese a sus ostensibles diferencias, bajo una misma FL - .

Es sobre este particular donde con más sentido se ha visto la implementación del carácter ontológico de la SM. La colocación es una relación sintagmática compleja en su naturaleza. Desde las "constelaciones" o "solidaridades" estructurales hasta los últimos acercamientos sobre esta llamativa coligación de unidades léxicas, se ha puesto de relieve un carácter experiencial subyacente a esta espontánea habilidad de asociar palabras. Es aquí, por tanto, donde puede que se comprenda en plenitud la elección de este corpus de ejemplos tan particular para la labor de descripción.

Se ha fomentado una sistematización que, por una parte, presentase a las FL como mecanismos formales, a fin de ganar en concreción y exhaustividad, y, por otra parte, al marco, para poder dar un sentido integrador a la gran variedad de esquemas que han podido registrarse.

Con todo, siempre hay margen de mejora para futuras investigaciones. En primer lugar, debemos aplicar nuestra propuesta de sistematización a un corpus de distinta naturaleza. Poder constatar que la metodología seguida es válida en el análisis de otros esquemas distintos al verbonominal, así como en otros inventarios colocacionales, como pueden ser las emociones, es imprescindible si se desea consolidar esta propuesta de trabajo. De igual forma, en el supuesto de que el modelo de sistematización resulte extrapolable a otros estudios, sería conveniente refinar ciertas bases teóricas. Algunas ampliaciones, como la extracción de los marcos directamente del español - y no de fuentes anglófonas - o la creación de un medio de representación más ilustrativo que las tablas - excesivamente informativas cuando la enumeración es extensa -, pueden suponer importantes progresos para los objetivos de detección, identificación y análisis que aquí se han planteado.

En conclusión, se ha proporcionado un planteamiento nuevo y concreto, con el que poder afrontar la colocación como un fenómeno cuyas motivaciones restan todavía sin identificar. En aplicación de dos modelos teóricos muy particulares, ha podido constatarse que se han cumplido los objetivos marcados y se han verificado las premisas formuladas en la hipótesis inicial.

\section{REFERENCIAS BIBLIOGRÁFICAS}

Barcelona, A. (2015). "Metonymy”. En Dabrowska, E. y D. Divjak (eds.). Handbook of Cognitive Linguistics. Berlin/Boston: De Gruyter Mouton, pp. 143-166.

Barrios Rodríguez, M. A. (2020). "The making of the Diretes dictionary". En Gavriilidou, Z., M. Mitsiaki y A. Fliatouras (eds.). Lexicography for inclusion. EURALEX Proceedings. XIX Congress 
of the European Association for Lexicography. Alexandroupolis: Democritus University of Thrace/ European Association for Lexicography, pp.13-22.

Bosque Muñoz, I. (2004). Redes: Diccionario combinatorio del español contemporáneo: las palabras en su contexto. Madrid: Ediciones SM.

Bouveret, M., con Ch. J. Fillmore (2008). "Matching verbo-nominal constructions in FrameNet with lexical functions in MTT". En Decesearis J. A. et al. (coord.). Proceedings of the XIII EURALEX International Congress (Barcelona, 15-19 July 2008), pp. 297-308.

Corpas Pastor, G. (2003). Diez años de investigación en fraseología: análisis sintáctico-semánticos, contrastivos y traductológicos. Madrid: Lingüística Iberoamericana.

Fillmore, C. J. (1976). "Frame semantics and the nature of language", Annals of the New York Academy of Sciences: Conference on the Origin and Development of Language and Speech, Vol. 280, pp. 20-32.

Fillmore, C. J. (1982). "Frame semantics". En Linguistic Society of Korea (ed.). Linguistics in the Morning Calm. Seoul: Linguistics Society of Korea, pp. 111-138.

Fillmore, C. J. (1985). "Frames and the semantics of understanding", Quaderni di Semantica, VI(2), pp. 222-254.

Mel'čuk, I. (1996) "Lexical functions: A tool for the description of lexical relations in a lexicon". En Wanner, L. (ed.), Lexical Functions in Lexicography and Natural Language Processing. Amsterdam/Philadelphia: John Benjamins, pp. 37-102.

Mel'čuk, I. (2006). "Colocaciones en el diccionario". En Alonso Ramos, $\mathrm{M}^{\mathrm{a}}$. (coord.). Diccionarios y fraseología. Anexos de Revista de Lexicografia, 3. A Coruña: Universidade da Coruña, pp. 11-44.

Mel'čuk, I. (2015a). Semantics from meaning to text. Vol. 3. Amsterdam/Philadelphia: John Benjamins.

Mel'čuk, I. (2015b). "Clichés, an Undestudied Subclass of Phrasemes", Yearbook of Phrasseology, 6(1), pp. 55-86.

Mel'čuk, I., A. Clas y A. Polguère (1995). Introduction à la lexicologie explicative et combinatoire. Louvain-la-Neuve : Édition Duculot.

Mel'čuk, I. y L. Wanner (1996). "Lexical Functions and Lexical Inheritance for Emotion Lexemes in German". En Wanner, L. (ed.), Lexical Functions in Lexicography and Natural Language Processing. Amsterdam/Philadelphia: John Benjamins, pp. 209-278.

Polguère, A. (2003). "Étiquetage sémantique des lexies dans la base des données DiCo", TAL. Traitement Automatique des Langues, Vol. 44, pp. 39-68.

Polguère, A. (2014). "From writing dictionaries to weaving lexical networks", International Journal of Lexicography, 27(4). Oxford University Press, pp. 396-418.

Rosch, E. H. (1978). "Principles of Categorization". En Rosch, E. y B. Lloyd (eds.). Cognition and categorization. Hillsdale: Lawrence Erlbaum, pp. 27-48. 